Check for updates

Cite this: RSC Adv., 2019, 9, 36742

\section{A salt-based method to adapt stiffness and biodegradability of porous collagen scaffolds $\uparrow$}

\author{
Luuk R. Versteegden, (D) a Marije Sloff, ${ }^{b}$ Henk R. Hoogenkamp, ${ }^{a}$ Michiel W. Pot, (D) a \\ Jeffrey Pang, (DD a Theo G. Hafmans, ${ }^{a}$ Thijs de Jong, (D) ${ }^{c}$ Theo H. Smit, (D) ${ }^{c}$ \\ Sander C. Leeuwenburgh, (D) ${ }^{d}$ Egbert Oosterwijk, ${ }^{b}$ Wout F. Feitz, ${ }^{b}$ \\ Willeke F. Daamen (D) $t^{\mathrm{a}}$ and Toin H. van Kuppevelt $\mathbb{D} t^{* a}$
}

Type I collagen scaffolds for tissue reconstruction often have impaired mechanical characteristics such as limited stiffness and lack of strength. In this study, a new technique is presented to fine-tune stiffness and biodegradability of collagen scaffolds by treatment with concentrated salt solutions. Collagen scaffolds were prepared by a casting, freezing and lyophilization process. Scaffolds were treated with $90 \%$ saturated salt solutions, the salts taken from the Hofmeister series, followed by chemical crosslinking. Treatment with salts consisting of a divalent cation in combination with a monovalent anion, e.g. $\mathrm{CaCl}_{2}$, resulted in fast shrinkage of the scaffolds up to approximately $10 \%$ of the original surface area. Effective salts were mostly at the chaotropic end of the Hofmeister series. Shrunken scaffolds were more than 10 times stiffer than non-shrunken control scaffolds, and displayed reduced pore sizes and swollen, less organized collagen fibrils. The effect could be pinpointed to the level of individual collagen molecules and indicates the shrinking effect to be driven by disruption of stabilizing hydrogen bonds within the triple helix. No calcium deposits remained in $\mathrm{CaCl}_{2}$ treated scaffolds. Subcutaneous implantation in rats showed similar biocompatibility compared to $\mathrm{H}_{2} \mathrm{O}$ and $\mathrm{NaCl}$ treated scaffolds, but reduced cellular influx and increased structural integrity without signs of major degradation after 3 months. In conclusion, high concentrations of chaotropic salts can be used to adjust the mechanical characteristics of collagen scaffolds without affecting biocompatibility. This technique may be used in regenerative medicine to stiffen collagen scaffolds to better comply with the surrounding tissues, but may also be applied for e.g.

slow release drug delivery systems.
Received 23rd August 2019 Accepted 26th October 2019 DOI: 10.1039/c9ra06651a

rsc.li/rsc-advances

\section{Introduction}

Type I collagen has been extensively used in the field of tissue engineering and regenerative medicine, particularly as a scaffolding material and as a controlled release system. ${ }^{1}$ Its excellent biocompatibility, widespread availability and straightforward processability make collagen a very useful biomaterial $^{2}$ that has been used in many (pre)clinical studies to

\footnotetext{
a Department of Biochemistry, Route 280, Radboud Institute for Molecular Life Sciences, Radboud university medical center, P. O. Box 9101, 6500 HB Nijmegen, The Netherlands. E-mail: toin.vankuppevelt@radbodumc.nl

${ }^{b}$ Department of Urology, Route 267, Radboud Institute for Molecular Life Sciences, Radboud university medical center, P. O. Box 9101, 6500 HB Nijmegen, The Netherlands

${ }^{c}$ Department of Medical Biology and Department of Orthopaedics, Amsterdam Movement Sciences, Amsterdam University Medical Centers, Meibergdreef 9, 1085AZ Amsterdam, The Netherlands

${ }^{d}$ Department of Dentistry, Route 309, Radboud university medical center, P. O. Box 9101, 6500 HB Nijmegen, The Netherlands

$\dagger$ Electronic supplementary information (ESI) available. See DOI: 10.1039/c9ra06651a

\$ These authors contributed equally to this work.
}

repair tissues like skin, ${ }^{3}$ cartilage ${ }^{4}$ and parts of the urogenital system. ${ }^{5}$ However, type I collagen scaffolds often show poor mechanical characteristics in applications where the scaffold is exposed to mechanical stress. ${ }^{6}$ When large defects are to be repaired, e.g. in diaphragmatic hernia, mechanical properties are generally insufficient. ${ }^{7}$

To strengthen collagen scaffolds, techniques such as chemical crosslinking using e.g. carbodiimide or glutaraldehyde are applied. $^{\mathbf{8 9}}$ Alternatively, components can be added to the collagen in order to enhance mechanical properties. Typically, synthetic polymers such as PLLA, ${ }^{10} \mathrm{PCL}^{11}$ or $\mathrm{PGA}^{12}$ have been used in this respect. However, addition of other components generally complicates the preparation process, may impair biocompatibility, and imposes additional regulatory constraints.

Increasing the concentration of insoluble type I collagen in a porous scaffold is another way to enhance mechanical stiffness. ${ }^{13}$ However, this procedure is poorly controllable for insoluble fibrillar collagen and maximum concentrations correspond to about $2 \%(\mathrm{w} / \mathrm{v})$ using current preparation techniques, which may still be insufficient for stiffer tissues. 
In this study, a method is presented that enables the construction of microporous collagen scaffolds with defined stiffness, that are biocompatible and display limited in vivo degradation for at least 3 months. The method is based on fast and extensive shrinkage of collagen scaffolds by treatment with highly concentrated salt solutions selected from the Hofmeister series of salts, a series based on the ability of anions and cations to alter protein stability, and to influence surface tension and solubility. ${ }^{\mathbf{1 4}}$ The method may expand the applications of collagen-based constructs in the regeneration of tissues with defined stiffness.

\section{Experimental section}

\section{Materials}

$\mathrm{N}$-Hydroxysuccinimide (NHS) was obtained from Fluka Chemie AG, Buchs, Switzerland. Acetic acid, ammonium chloride $\left(\mathrm{NH}_{4} \mathrm{Cl}\right)$, ammonium sulfate $\left(\left(\mathrm{NH}_{4}\right)_{2} \mathrm{SO}_{4}\right)$, bovine serum albumin, 1-ethyl-3-(3-dimethylaminopropyl)carbodiimide (EDC), ethanol, glutaraldehyde, hydrogen chloride, nitric acid, paraformaldehyde, sodium chloride $(\mathrm{NaCl})$, sodium hydrogen phosphate $\left(\mathrm{Na}_{2} \mathrm{HPO}_{4}\right)$ and sodium sulfate $\left(\mathrm{Na}_{2} \mathrm{SO}_{4}\right)$ were from Merck, Darmstadt, Germany. Glycine was from Scharlau Chemicals, Barcelona, Spain. Barium perchlorate $\left(\mathrm{Ba}\left(\mathrm{ClO}_{4}\right)_{2}\right)$, barium sulfate $\left(\mathrm{BaSO}_{4}\right)$, calcium chloride $\left(\mathrm{CaCl}_{2}\right)$, calcium sulfate $\left(\mathrm{CaSO}_{4}\right)$, magnesium chloride $\left(\mathrm{MgCl}_{2}\right)$, magnesium perchlorate $\left(\mathrm{Mg}\left(\mathrm{ClO}_{4}\right)_{2}\right)$, 2-( $\mathrm{N}$-morpholino)ethane sulfonic acid (MES) and trinitrobenzene sulfonic acid (TNBS) were from Sigma Aldrich, St. Louis, MO, USA. Barium chloride $\left(\mathrm{BaCl}_{2}\right)$ and magnesium sulfate $\left(\mathrm{MgSO}_{4}\right)$ were from VWR International, Radnor, PA, USA.

\section{Preparation of shrunken collagen scaffolds}

(1) Porous scaffold preparation. Type I collagen fibrils were purified from bovine achilles tendon using extractions steps with aqueous solutions of $\mathrm{NaCl}$, urea, diluted acetic acid, acetone and demineralized water. ${ }^{15}$ Purified collagen was suspended in $0.25 \mathrm{M}$ acetic acid $(0.8 \% \mathrm{w} / \mathrm{v})$, swollen overnight, and subsequently homogenized using a Silverson L5M-A laboratory mixer (Silverson, Chesham, UK) by mixing for $6 \mathrm{~min}$ at $2500 \mathrm{rpm}$ (3 min with general purpose disintegrating work head and $3 \mathrm{~min}$ with a slotted work head). Next, the suspension was deaerated using centrifugation at $100 \mathrm{~g}$ for $30 \mathrm{~min}$. The suspension was poured into 12 -well plates $(1.5 \mathrm{~mL})$ and 6-well plates $(4 \mathrm{~mL})$, frozen for $4 \mathrm{~h}$ at $-20{ }^{\circ} \mathrm{C}$ on an aluminum surface for optimal thermal conduction and lyophilized (Zirbus sublimator 500II, Bad Grund, Germany).

(2) Shrinkage of scaffolds by Hofmeister series of salt treatment. To analyze the effect of different concentrated salt solutions, non-crosslinked porous scaffolds $(\varnothing 22 \mathrm{~mm})$ were exposed to $4 \mathrm{~mL}$ of $90 \%$ saturated salt solutions in demineralized water. Twelve salts were selected from the entire spectrum of the Hofmeister series (see ESI Table $1 \dagger$ ). To enhance penetration of the salt solution into the scaffold, the surface tension of $4 \mathrm{~mL} 90 \%$ saturated salt solutions was reduced by the addition of $10 \mu \mathrm{L} 100 \%(\mathrm{v} / \mathrm{v})$ ethanol. As a control, scaffolds were incubated in demineralized water with $10 \mu \mathrm{L}$ ethanol. After $48 \mathrm{~h}$, the surface of the scaffolds was scanned (Epson V750 PRO) and the surface area was measured using image analysis software (ImageJ version 1.47i, NIH, Bethesda, MD, USA). For the salts that showed high shrinkage (more than 90\% compared to original surface), the shrinkage was determined at different concentrations. All experiments were performed three times independently.

(3) Shrinkage process for $\mathbf{C a C l}_{2}$. We focused on $\mathrm{CaCl}_{2}$, as it proved to be one of the most effective salts (see Results section). As controls, scaffolds were treated with $\mathrm{NaCl}$ (salt treatment, but no shrinkage) and demineralized water $\left(\mathrm{H}_{2} \mathrm{O}\right)$. Scaffolds were shrunken in an ascending series of $\mathrm{CaCl}_{2}$ to achieve gradual shrinkage. Briefly, they were first incubated for $15 \mathrm{~min}$ in $1 \mathrm{M}$ $\mathrm{CaCl}_{2}$ or $1 \mathrm{M} \mathrm{NaCl}$, followed by $15 \mathrm{~min} 2 \mathrm{M}$, and $30 \mathrm{~min} 4 \mathrm{M}$ salt solution. Control scaffolds were incubated for $30 \mathrm{~min}$ in demineralized water. Macroscopic images were taken and videos of the shrinking process with $\mathrm{CaCl}_{2}$ were recorded with a Sony Cyber-shot DSC-H10 (Sony, Minato, Tokyo, Japan).

(4) Stabilization of scaffolds by chemical crosslinking. After incubation in $4 \mathrm{M}$ salt solution or demineralized water, scaffolds were immediately crosslinked using a carbodiimide zero-length crosslinker. In brief, scaffolds were incubated in $4 \mathrm{~mL} 50 \mathrm{mM}$ MES buffer, pH 5.0, containing 40\% (v/v) ethanol, $33 \mathrm{mM}$ EDC and $6 \mathrm{mM}$ NHS for $3 \mathrm{~h}$. Next, the scaffolds were extensively washed 6 times with demineralized water to remove residual crosslinking reagents and salt. Non-crosslinked scaffolds were also washed 6 times with demineralized water. Finally, scaffolds were placed in $70 \%(\mathrm{v} / \mathrm{v})$ ethanol and stored at $-20{ }^{\circ} \mathrm{C}$ until use.

(5) Sterilization of the scaffolds. Scaffolds used for in vivo evaluation were sterilized in PBS using gamma irradiation at 25 kGy from a ${ }^{60}$ Co source (ISO 9001; Synergy Health BV, Ede, The Netherlands). Before sterilization, $\varnothing 6 \mathrm{~mm}$ discs were cut from the scaffolds after which they were washed 6 times with PBS to remove ethanol.

\section{Scaffold characterization}

(1) Scanning electron microscopy. For scanning electron microscopy, crosslinked scaffolds were washed in demineralized water to remove storage medium, and subsequently frozen at $-80{ }^{\circ} \mathrm{C}$. The frozen scaffolds were lyophilized, mounted on stubs with double-sided carbon tape, and coated with an ultrathin gold layer (Scancoat Six Sputter Coater, Edwards, Crawley, United Kingdom). The samples were evaluated with a Sigma 300 field emission scanning electron microscope (Carl Zeiss B. V., Sliedrecht, The Netherlands) with an accelerating voltage of $10 \mathrm{kV}$. The pore sizes of the different scaffolds were determined using Image analysis software.

(2) Transmission electron microscopy. Scaffolds were fixed in $2 \%(\mathrm{w} / \mathrm{v})$ glutaraldehyde in $0.1 \mathrm{M}$ phosphate buffer (PB), $\mathrm{pH}$ 7.3 for $24 \mathrm{~h}$ at $4{ }^{\circ} \mathrm{C}$. After washing steps in $\mathrm{PB}$, scaffolds were treated with $1 \%$ osmium tetroxide in $0.1 \mathrm{M}$ PB for $1 \mathrm{~h}$. Next, scaffolds were washed in $\mathrm{PB}$, dehydrated in graded series of alcohol and embedded in Epon 812 . Sections $(1 \mu \mathrm{m})$ were cut, stained with toluidine blue and examined with a light- 
microscope (Dialux 20, Leitz). Ultrathin sections $60 \mathrm{~nm}$ were cut, followed by treatment with uranyl-acetate/lead-citrate for double contrast. Sections were imaged using a transmission electron microscope (JEOL 1010).

(3) Determination of stiffness. The stiffness of $\mathrm{CaCl}_{2}, \mathrm{NaCl}$ and $\mathrm{H}_{2} \mathrm{O}$ treated collagen scaffolds were measured with the PIUMA nano-indenter (Optics11, Amsterdam, The Netherlands; Fig. 3A). ${ }^{16}$ Before the measurement, scaffolds were incubated in PBS for $30 \mathrm{~min}$ followed by a passivation step of $60 \mathrm{~min}$ in $5 \%$ bovine serum albumin in PBS to prevent adhesion of the probe to the scaffolds. Per sample, 25 indentions were performed in a grid of $1 \times 1 \mathrm{~mm}$ with a distance of $200 \mu \mathrm{m}$ between individual indentations (Fig. 3B). For soft samples, an indenter probe with a stiffness of $0.05 \mathrm{~N} \mathrm{~m}^{-1}$ was used and for stiffer samples a probe with a stiffness of $0.47 \mathrm{~N} \mathrm{~m}^{-1}$. Both probes had a tip diameter of $180 \mu \mathrm{m}$. The indentation depth, reached with a speed of $5 \mu \mathrm{m} \mathrm{s}^{-1}$, was $15 \mu \mathrm{m}$ and the probe was kept in place for $2 \mathrm{~s}$ (indentation time). Each scaffold type was measured 3 times independently.

(4) Calcium analysis of $\mathrm{CaCl}_{2}$ treated scaffolds. Two distinct methodologies were used to determine potential remnants of the $\mathrm{CaCl}_{2}$ shrinking treatment. Inductively coupled plasma mass spectrometry (ICP-MS) was performed to detect elemental calcium, and Fourier transform infrared spectroscopy (FTIR) was performed to detect the presence of calciumcontaining compounds which may have been formed after $\mathrm{CaCl}_{2}$ treatment. For the ICP-MS measurement, scaffolds were first hydrolyzed with $50 \%(\mathrm{v} / \mathrm{v})$ nitric acid and subsequently diluted to a final concentration of $0.05 \mathrm{mg} \mathrm{mL}^{-1}$ in $1 \%$ nitric acid. Samples were analyzed using a X series I - ICP-MS (Thermo Fisher Scientific, Waltham, MA, USA). The total calcium content was calculated based on the measurement of the ${ }^{43} \mathrm{Ca}$ isotope as ${ }^{40} \mathrm{Ca}$ interfered with other elements. Oneway ANOVA with Bonferroni post-hoc testing was performed to determine statistical differences. Fourier Transform Infrared Spectroscopy (FTIR, Spectrum Two, PerkinElmer, Waltham, MA, USA) was used to study the presence of calcium-containing compounds in the scaffolds. The FTIR spectra were measured in Attenuated Total Reflectance mode, with a spectral resolution of $4 \mathrm{~cm}^{-1}$, a scan speed of $0.2 \mathrm{~cm} \mathrm{~s}^{-1}$ and 10 scans per measurement.

(5) Differential Scanning Calorimetry. Differential scanning calorimetry (DSC Q1000, TA Instruments, New Castle, DE, USA) was used to assess potential denaturation of type I collagen caused by the salt treatment by measuring the denaturation temperature of non-crosslinked scaffolds treated with $\mathrm{CaCl}_{2}, \mathrm{NaCl}$ and $\mathrm{H}_{2} \mathrm{O}$. Purified insoluble type I collagen (starting material for scaffold preparation) was used as a control. Samples $(\sim 1 \mathrm{mg})$ in aluminium pans with $25 \mu \mathrm{L}$ PBS were heated from 1 to $80{ }^{\circ} \mathrm{C}$ with a rate of $5^{\circ} \mathrm{C} \mathrm{min}^{-1}$. The endothermic peak was determined using TA Instruments Universal Analysis 2000 software (Version 4.5 A).

(6) Gel electrophoresis. Sodium dodecyl sulfate polyacrylamide gel electrophoresis (SDS-PAGE) was performed to assess potential denaturation of collagen in the scaffold after salt treatment. Briefly, 2-3 $\mathrm{mg}$ of non-crosslinked collagen scaffolds was digested overnight at ambient temperature in 75 $\mu \mathrm{L} 0.25 \mathrm{M}$ acetic acid containing $3 \mathrm{mg} \mathrm{mL}^{-1}$ pepsin after which an equal volume of a 2 -fold concentrated non-reducing sample buffer was added. Samples and controls were loaded on an $8 \%$ $(\mathrm{w} / \mathrm{v})$ polyacrylamide gel without prior heating. After running at $150 \mathrm{~V}$, the gel was stained with $0.1 \%(\mathrm{w} / \mathrm{v})$ Coomassie Brilliant Blue solution.

(7) Evaluation of degree of crosslinking. The degree of crosslinking of the salt treated scaffolds was calculated by assaying the loss of primary amine groups after crosslinking using a TNBS assay. ${ }^{17}$ After salt treatment, crosslinking and rinsing steps with water, the scaffolds were lyophilized and subsequently incubated for $30 \mathrm{~min}$ in $4 \%(\mathrm{w} / \mathrm{v}) \mathrm{Na}_{2} \mathrm{HPO}_{4}$ at $22{ }^{\circ} \mathrm{C}$. Subsequently, samples were incubated for $2 \mathrm{~h}$ at $40{ }^{\circ} \mathrm{C}$ in $0.5 \%(\mathrm{w} / \mathrm{v})$ TNBS in demineralized water, after which the samples were hydrolyzed with $6 \mathrm{M} \mathrm{HCl}$ for $1.5 \mathrm{~h}$ at $60{ }^{\circ} \mathrm{C}$. Absorbance at $420 \mathrm{~nm}$ was measured with a spectrophotometer (Bio-Tek, Bad Friedrichshall, Germany). Glycine was used for the calibration curve. Non-crosslinked controls for each condition were used to calculate the percentage of crosslinked amine groups. Experiments were performed in triplicate in three different experiments.

\section{In vivo evaluation of salt treated scaffolds in a rat model}

The study was approved by the Ethical Committee on Animal Research of the Radboud University Nijmegen, The Netherlands (RU-DEC 2013-144) and executed according to Dutch legislation and guidelines for the use of laboratory animals. Nine male Wistar rats (Harlan Laboratories, Horst, The Netherlands) of 3 months old ( $\sim 400 \mathrm{~g}$ ) were housed in trios in a controlled environment (19-21 ${ }^{\circ} \mathrm{C}, 12-12 \mathrm{~h}$ day per night cycle) with ad libitum access to food and water. Using Microsoft Excel, the rats were randomly divided over three experimental groups. Rats were anesthetized with $2-3 \%(\mathrm{v} / \mathrm{v})$ isoflurane, and received buprenorphine by intramuscular injection as analgesic. Three different scaffolds (a $\mathrm{CaCl}_{2}$ treated scaffold, a $\mathrm{NaCl}$ treated scaffold, and $\mathrm{a}_{2} \mathrm{O}$ treated scaffold) were randomly implanted in subcutaneous pockets created on the back of each rat. At the time of implantation, researchers performing evaluations were blinded for the type of scaffold by naming them A, B and C. Reference sutures (Prolene 5.0, Ethicon, Somerville, NJ) were placed next to the scaffold. Wounds were sutured using Prolene 5.0 and for reinforcement of the sutures hog rings were placed over the wound. Immediately after surgery and 12, 24, 36 and $48 \mathrm{~h}$ later, rats received additional buprenorphine via intramuscular injection. During the whole experiments the general health condition of the rats was monitored. At 3 days (group 1), 14 days (group 2) and 90 days (group 3) after implantation the animals were sacrificed using carbon dioxide gas. Scaffolds were excised from the subcutaneous pockets, fixated using $4 \%$ (w/v) paraformaldehyde in phosphate buffer ( $\mathrm{pH}$ 7.4) and embedded in paraffin. Sections $(5 \mu \mathrm{m})$ were obtained using a HM 340E Electronic Rotary Microtome (Thermo Fisher Scientific, Waltham, MA, USA). Tissue sections were stained with haematoxylin/eosin and elastin Von Masson. To visualize calcifications, a von Kossa and an Alizarin red staining were performed. Blinding was applied for the animal experiments; 
however, at time of evaluation the blinded feature of this study was lost due to the distinct characteristics of each scaffold type. Haematoxylin and eosin stained sections were scored semi quantitatively from 0 (not present) to 3 (abundantly present), where sporadic events were scored as 0.1. Statistical significance was determined using an Independent Samples Kruskal Wallistest for comparison between scaffolds at the three time points. Differences were considered statistically significant when $p<$ 0.05 .

\section{Results}

To evaluate the effect of the Hofmeister salts on porous discshaped collagen scaffolds, scaffolds were incubated for $48 \mathrm{~h}$ in $90 \%$ saturated solutions. For a number of salts (e.g. $\left.\mathrm{CaCl}_{2}\right)$, major shrinkage of the scaffolds was observed, whereas for other salts intermediate, minor or no shrinkage was noticed (Fig. 1). The surface area was shrunken for more than $90 \%$ in case of $\mathrm{CaCl}_{2}$ (93 $\pm 1 \%$, Fig. $\left.1 \mathrm{~A}\right), \mathrm{Ba}\left(\mathrm{ClO}_{4}\right)_{2}(91 \pm 1 \%), \mathrm{MgCl}_{2}(93$ $\pm 1 \%$ ), and $\mathrm{Mg}\left(\mathrm{ClO}_{4}\right)_{2}(94 \pm 1 \%)$ (Fig. 1B). The process was

A
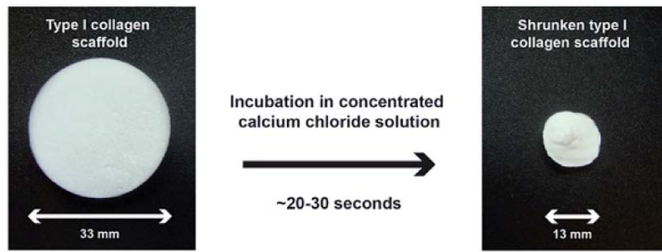

B

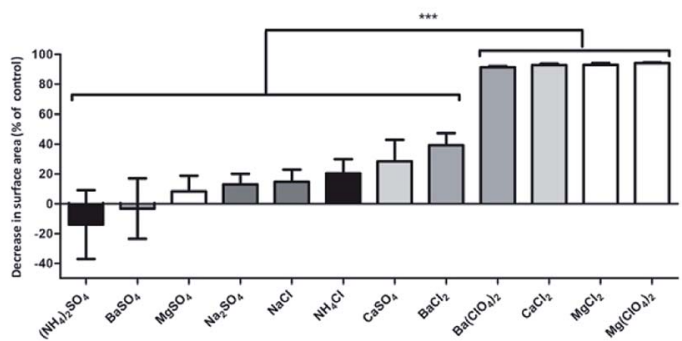

C

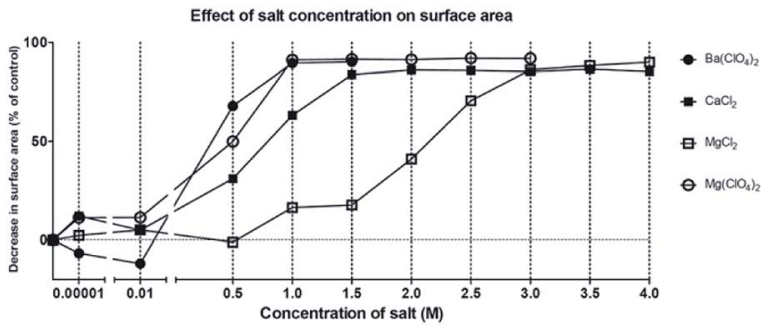

Fig. 1 The shrinkage effect of different salts from the Hofmeister series on $0.8 \%(\mathrm{w} / \mathrm{v})$ collagen scaffolds. (A) Type I collagen scaffold before and after exposure to $4 \mathrm{M} \mathrm{CaCl}_{2}$. (B) The effect of $90 \%$ saturated salt solutions ( $x$-axis) on the surface area of on collagen scaffolds ( $y$ axis). Note that $\mathrm{Ba}\left(\mathrm{ClO}_{4}\right)_{2}, \mathrm{CaCl}_{2}, \mathrm{MgCl}_{2}$, and $\mathrm{Mg}\left(\mathrm{ClO}_{4}\right)_{2}$ induced shrinkage to over $90 \%$ compared to control scaffolds, $n=3$. Statistical analysis: one-way ANOVA with Bonferroni post-hoc test, $p<0.001$. Due to variabilities in solubility, the $90 \%$ saturated salt solutions vary in molarity (see ESI Table $1+$ ). (C) The effect of salt concentration ( $x$-axis) on the degree of shrinkage ( $y$-axis) for a subset of salts that showed over $90 \%$ shrinkage in (B), $n=3$. extremely fast: within $30 \mathrm{~s}$ the scaffold had almost completely shrunken (Movie $1 \dagger$ ). Salts that caused only limited shrinkage were $\mathrm{MgSO}_{4}(8 \pm 11 \%), \mathrm{Na}_{2} \mathrm{SO}_{4}(13 \pm 7 \%)$, NaCl (15 $\pm 8 \%$ ), $\mathrm{NH}_{4} \mathrm{Cl}(20 \pm 9 \%), \mathrm{CaSO}_{4}(28 \pm 14 \%)$, and $\mathrm{BaCl}_{2}(39 \pm 8 \%)$. $\left(\mathrm{NH}_{4}\right)_{2} \mathrm{SO}_{4}$ and $\mathrm{BaSO}_{4}$ displayed no shrinkage. In general, salts containing divalent cations in combination with monovalent anions, resulted in major shrinkage of the scaffold.

Focussing on salts that induced shrinkage of $>90 \%$, we studied the minimal concentration required to achieve full shrinkage of the scaffold (Fig. 1C). For $\mathrm{Mg}\left(\mathrm{ClO}_{4}\right)_{2}$ and $\mathrm{Ba}\left(\mathrm{ClO}_{4}\right)_{2}$ $1 \mathrm{M}$ was needed, whereas for $\mathrm{CaCl}_{2}$ and $\mathrm{MgCl}_{2} 1.5$ and $3 \mathrm{M}$ were required. Being one of the most effective salts for shrinkage, $\mathrm{CaCl}_{2}$ was selected for further studies, comparing this salt treatment to treatment using $\mathrm{NaCl}$ and $\mathrm{H}_{2} \mathrm{O}$.

Scaffolds treated with $\mathrm{CaCl}_{2}, \mathrm{NaCl}$ and $\mathrm{H}_{2} \mathrm{O}$ were characterized by scanning and transmission electron microscopy. Crosssectional images revealed that salt treated scaffolds remained porous (Fig. 2, left panels). $\mathrm{CaCl}_{2}$ treated scaffolds displayed circular pores but, compared to the $\mathrm{H}_{2} \mathrm{O}$ treated scaffold, the pore size decreased from $40-120 \mu \mathrm{m}$ to $20-60 \mu \mathrm{m}$. In the $\mathrm{NaCl}$ treated scaffolds, the pore structure had somewhat flattened and the size of pores was slightly decreased. $\mathrm{CaCl}_{2}$ treatment had a distinct swelling effect on the individual collagen fibrils in the scaffolds. The fibrils in $\mathrm{CaCl}_{2}$ treated scaffolds appeared distended (Fig. 2B, right panels), resulting in the loss of the characteristic banding pattern, whereas clear banding patterns were visible in $\mathrm{NaCl}$ and $\mathrm{H}_{2} \mathrm{O}$ treated scaffolds (Fig. $2 \mathrm{~B}$ and $\mathrm{C}$, right panels).

The shrinking process may have an effect on the mechanical properties of the scaffolds due to the increased collagen content per volume. Therefore, the stiffness was assessed using nanoindentation (Fig. 3). $\mathrm{CaCl}_{2}, \mathrm{NaCl}$ and $\mathrm{H}_{2} \mathrm{O}$ treated scaffolds were measured three times independently with 25 indentations on different locations per scaffold (Fig. 3B). $\mathrm{CaCl}_{2}$ scaffolds $(7.4 \times$ $\left.10^{3} \mathrm{~Pa}\right)$ were stiffer compared to the $\mathrm{NaCl}\left(1.2 \times 10^{3} \mathrm{~Pa}\right)$ and the $\mathrm{H}_{2} \mathrm{O}$ treated scaffolds $\left(4.4 \times 10^{2} \mathrm{~Pa}\right)$ as shown in (Fig. 3C). Peak values showed a stiffness of more than $3.0 \times 10^{4} \mathrm{~Pa}$, most likely when the tip indented exactly on top of collagen structure in the porous scaffold indicating that the stiffness of $\mathrm{CaCl}_{2}$ treated collagen material is higher than $7.4 \times 10^{3} \mathrm{~Pa}$.

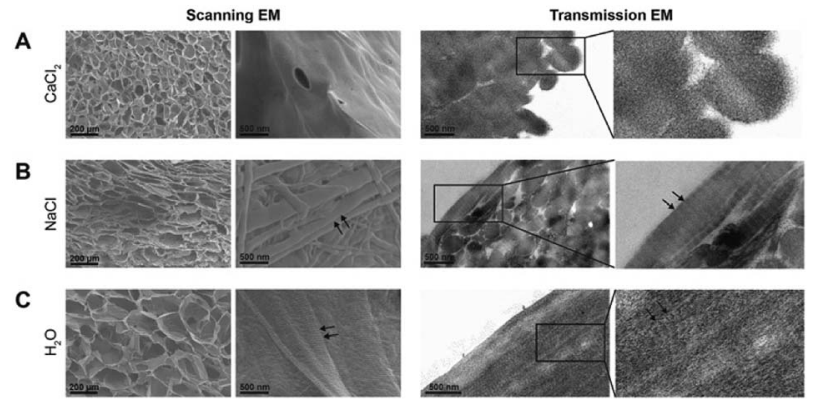

Fig. 2 Morphological characterization of collagen scaffolds. Scanning and transmission electron microscopical images of $(\mathrm{A}) \mathrm{a} \mathrm{CaCl}_{2}$ treated scaffold showing the altered pore structure and the swollen fibrils without banding pattern; ( $B$ and $C$ ) $\mathrm{NaCl}(B)$ and $\mathrm{H}_{2} \mathrm{O}$ (C) treated scaffolds showing the characteristic striation of collagen fibrils indicated with black arrows. 
A
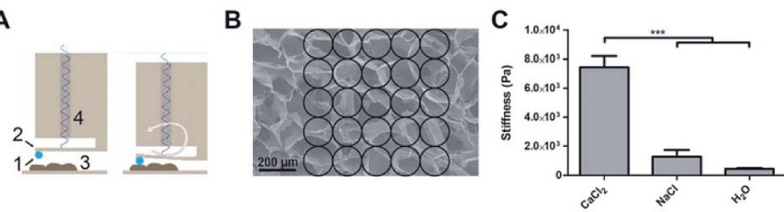

Fig. 3 Mechanical characterization using nanoindentation. (A) Working mechanism of PIUMA nanoindentation equipment: a probe (1) connected to a cantilever (2) indents the sample (3) and bends through. The degree of bending is measured via an optical cable (4) and converted to the degree of stiffness. (B) Locations of indentation on the scaffold visualized with a representative SEM image. (C) The stiffness of $\mathrm{CaCl}_{2}, \mathrm{NaCl}$ and $\mathrm{H}_{2} \mathrm{O}$ treated scaffolds measured with a PIUMA nanoindentation apparatus. Each condition was tested in triplicate with 25 indentations per scaffold. Bars represent mean \pm standard error of the mean. One-way ANOVA with Bonferroni posthoc test, $* * * p<0.0001$.

Next, we assessed the presence of calcium or calciumcontaining compounds in collagen scaffolds. Inductively coupled plasma mass spectrometry showed that the $\mathrm{CaCl}_{2}$ treated scaffold contained only $17.8 \pm 5.2 \mathrm{ppb}$ calcium corresponding to $0.36 \pm 0.03 \mathrm{ng}$ per $\mu \mathrm{g}$ scaffold (Table 1). The $\mathrm{NaCl}$ and $\mathrm{H}_{2} \mathrm{O}$ treated scaffolds contained $0.25 \pm 0.02$ and $0.29 \pm 0.1$ $\mathrm{ng}$ calcium per $\mu \mathrm{g}$ scaffold respectively, which were both not significantly different from the $\mathrm{CaCl}_{2}$ treated scaffold (one-way ANOVA with Bonferroni post-hoc testing, $p>0.05$ ). This suggests that no remnants of the calcium treatment were present in the $\mathrm{CaCl}_{2}$ shrunken scaffolds. Using Fouriertransform infrared spectroscopy no absorption peaks were detected that could be attributed to calcium-containing compounds (Table 1 ).

The degree of crosslinking in the $\mathrm{CaCl}_{2}, \mathrm{NaCl}$, and $\mathrm{H}_{2} \mathrm{O}$ treated scaffolds was estimated by the loss of primary amine groups (Fig. 4). Non-crosslinked $\mathrm{CaCl}_{2}, \mathrm{NaCl}$, and $\mathrm{H}_{2} \mathrm{O}$ treated scaffolds contained $241 \pm 41,244 \pm 43,246 \pm 37 \mathrm{nmol}$ of primary amine groups per $\mathrm{mg}$ collagen, respectively, whereas crosslinked scaffolds had $81 \pm 12,111 \pm 8$ and $139 \pm 10 \mathrm{nmol}$. The reduction of primary amine groups was $66 \%$ for the $\mathrm{CaCl}_{2}$ treated scaffold, $55 \%$ for the $\mathrm{NaCl}$ treated scaffold and $42 \%$ for the $\mathrm{H}_{2} \mathrm{O}$ treated scaffold (Fig. 4A). The difference in crosslinking percentage between the three scaffolds types were statistically different as assessed by a one-way ANOVA with Bonferroni posthoc testing, $p<0.05$.

The extent of denaturation was assessed using electron microscopy and SDS-PAGE. Electron microscopical images of $\mathrm{CaCl}_{2}$ treated scaffolds (non-EDC/NHS crosslinked) displayed swollen fibrils which may be caused by partial denaturation
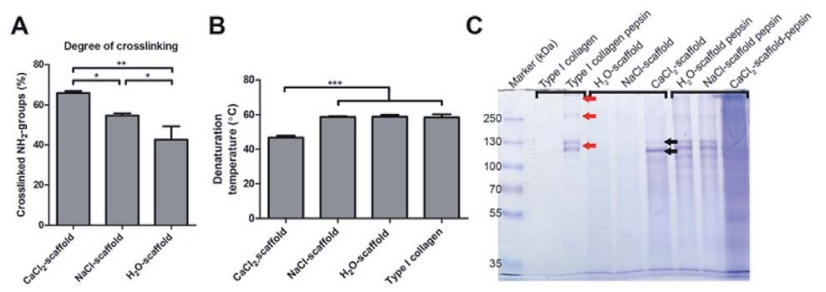

Fig. 4 Biochemical and biophysical characterization of $\mathrm{CaCl}_{2}, \mathrm{NaCl}$ and $\mathrm{H}_{2} \mathrm{O}$ treated scaffolds. (A) Degree of crosslinking (TNBS-assay) indicating differences between scaffolds ( $n=3$, one-way ANOVA with Bonferroni post-hoc test, $* p<0.05, * * p<0.01$ ). (B) Denaturation temperature $\left(T_{\mathrm{d}}\right)$ of purified insoluble type I collagen and various scaffold types indicated that $\mathrm{CaCl}_{2}$ treatment lowered the $T_{\mathrm{d}}(n=6$, one-way ANOVA with Bonferroni post-hoc test, $* * * P<0.001$ ). (C) SDS-PAGE gel of purified insoluble type I collagen, $\mathrm{CaCl}_{2}, \mathrm{NaCl}$ and $\mathrm{H}_{2} \mathrm{O}$ treated scaffolds with and without pepsin treatment indicating that $\mathrm{CaCl}_{2}$ treatment causes denaturation of the triple helix. The red arrows indicate the location of $\alpha, \beta$, and $\gamma$ bands after pepsin digestion and the black arrows indicates the location of $\alpha$ bands after $\mathrm{CaCl}_{2}$ treatment.

induced by $\mathrm{CaCl}_{2}$. Differential scanning calorimetry showed that the denaturation temperature was reduced from $58 \pm 2{ }^{\circ} \mathrm{C}$ (type I collagen) to $47 \pm 1{ }^{\circ} \mathrm{C}$ after calcium treatment indicating denaturation (Fig. 4B). The denaturation temperature of the $\mathrm{H}_{2} \mathrm{O}\left(59 \pm 1{ }^{\circ} \mathrm{C}\right)$ and $\mathrm{NaCl}\left(59 \pm 1{ }^{\circ} \mathrm{C}\right)$ treated scaffolds did not change significantly from purified insoluble type I collagen. SDS-PAGE substantiated that $\mathrm{CaCl}_{2}$ treatment induced denaturation (Fig. 4C). No bands will be visible on an SDS-PAGE gel when insoluble type I collagen is in its native state, as shown by the control samples $\left(\mathrm{H}_{2} \mathrm{O}\right.$ and $\mathrm{NaCl}$ treated scaffolds). The $\mathrm{CaCl}_{2}$ treated collagen showed clear collagen $\alpha-$ chain bands (black arrows) indicating denaturation of the insoluble type I collagen fibrils. When scaffolds were subjected to pepsin treatment (liberating collagen molecules from fibrils), $\alpha, \beta$, and $\gamma$ bands were detected for $\mathrm{H}_{2} \mathrm{O}$ and $\mathrm{NaCl}$ treated scaffolds (see red arrows in Fig. 4C), while for $\mathrm{CaCl}_{2}$ treated scaffolds a smear was observed. Pepsin does not cleave intact type I collagen triple helices, but if the helix is partially denaturated it becomes susceptible for pepsin cleavage over the entire helix, resulting in a smear on gel. Consequently, the results of the SDS-PAGE indicate $\mathrm{CaCl}_{2}$ induced denaturation of the triple helix.

Finally, we performed an in vivo biocompatibility study upon subcutaneous implantation in rats. All rats survived surgery without complications and did not exhibit any discomfort caused by the subcutaneously implanted scaffolds throughout the entire study. The surgical wounds healed normally. Two

Table 1 Presence of calcium in treated scaffolds

\begin{tabular}{lll}
\hline Scaffold type & $\begin{array}{l}\text { ICP-MS calcium content } \\
\text { (ng calcium per } \mu \text { g collagen scaffold) }\end{array}$ & $\begin{array}{l}\text { FT-IR presence of } \\
\text { calcium-containing compounds }\end{array}$ \\
\hline $\mathrm{CaCl}_{2}$ scaffold & $0.36 \pm 0.03$ & None \\
$\mathrm{NaCl}^{2}$ saffold & $0.25 \pm 0.02$ & None \\
$\mathrm{H}_{2} \mathrm{O}$ scaffold & $0.29 \pm 0.1$ & None
\end{tabular}


scaffolds could not be retrieved upon explantation; i.e. 1 untreated scaffold at day 3 and $1 \mathrm{NaCl}$ treated scaffold at day 14 . Three days after implantation the original shape of all scaffolds was maintained, and they were covered with a macroscopically visible layer of fibrous tissue. Visual signs of inflammation were absent, but histologically a minor immune response was observed for all scaffolds based on the presence of granulocytes and macrophages/giant cells. For the $\mathrm{CaCl}_{2}$ treated scaffolds, these cells were located at the rims of the scaffold (ESI Fig. $1 \dagger$ ). At day 14 after implantation, all scaffolds were partially encapsulated, but the $\mathrm{H}_{2} \mathrm{O}$ and $\mathrm{NaCl}$ treated scaffolds had clearly lost their original shape, while this was not macroscopically observed for $\mathrm{CaCl}_{2}$ treated scaffolds. Microscopic analysis showed a somewhat increased cellular infiltration in $\mathrm{H}_{2} \mathrm{O}$ and $\mathrm{NaCl}$ treated scaffolds, which may have contributed to the deformation and biodegradation of the scaffold (ESI Fig. $1 \dagger$ ). At day 90 days after implantation, scaffolds treated with $\mathrm{H}_{2} \mathrm{O}$ and $\mathrm{NaCl}$ showed clear bio-degradation, whereas the $\mathrm{CaCl}_{2}$ treated scaffolds still kept their original shape. Statistical analysis revealed differences in cellular influx, degradation and presence of blood vessels between scaffolds at this time points (Table 2). All scaffolds were fully encapsulated as observed in the H\&E staining in Fig. 5. H\&E and Elastin von Masson (EvM) stainings indicated that cells (giant cells, monocytes, macrophages and fibroblasts) infiltrated the $\mathrm{H}_{2} \mathrm{O}$ and $\mathrm{NaCl}$ treated scaffolds (Fig. 5B and C, left and middle panel). Cell migration was limited for the $\mathrm{CaCl}_{2}$ treated scaffolds as cells were only observed at the outer edges of the scaffold (Fig. 5A, left and middle panel). For all scaffolds the immunological reaction was mild. Alizarin Red and von Kossa stainings did not reveal any

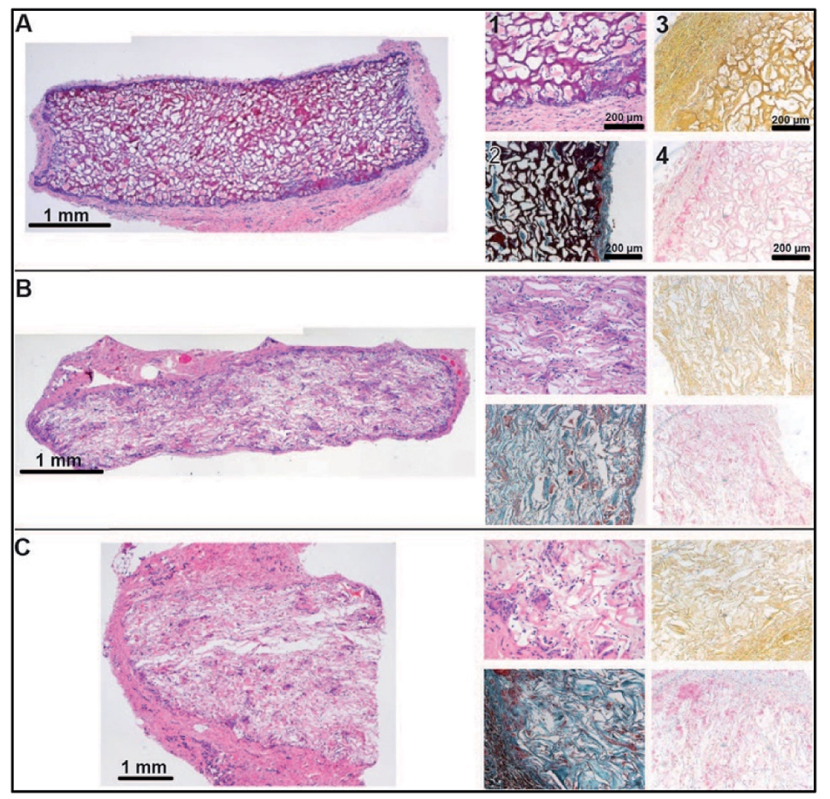

Fig. 5 Biocompatibility of $\mathrm{CaCl}_{2}, \mathrm{NaCl}$, and $\mathrm{H}_{2} \mathrm{O}$ treated scaffolds study in a subcutaneous rat model. In the left panel H\&E overview images are depicted of a $\mathrm{CaCl}_{2}$ treated scaffold (A), a $\mathrm{NaCl}$ treated scaffold (B), and $\mathrm{a}_{2} \mathrm{O}$ treated scaffold (C) 90 days after implantation. On the right, enlarged images of similar magnification are shown for every scaffold type. Image (1) H\&E staining, (2) Elastin von Masson staining, (3) Alizarin Red staining, (4) von Kossa staining.

calcium/calcification in the implanted scaffolds (Fig. 5C, right panels). See ESI Fig. $2 \uparrow$ for positive controls for calcium deposits (human bone tissue).

Table 2 Semi quantitative scores of haematoxylin and eosin stained sections of subcutaneously implanted collagen scaffolds treated with salt

\begin{tabular}{|c|c|c|c|c|}
\hline Parameter & Scaffold & Day 3 & Day 14 & Day 90 \\
\hline & $\mathrm{NaCl}$ & $0.3 \pm 0.3$ & $2.3 \pm 0.4$ & $1.7 \pm 0.6$ \\
\hline & Untreated & $1.0 \pm 0.7$ & $2.5 \pm 0.5$ & $1.7 \pm 1.3$ \\
\hline Cellular influx & $\mathrm{CaCl}_{2}$ & $0.0 \pm 0.0^{a}$ & $0.5 \pm 0.5$ & $0.7 \pm 0.3^{a}$ \\
\hline \multirow[t]{3}{*}{ Granulocytes } & $\mathrm{CaCl}_{2}$ & $1.2 \pm 0.6$ & $0.1 \pm 0.0$ & $0.1 \pm 0.0$ \\
\hline & $\mathrm{NaCl}$ & $0.5 \pm 0.0$ & $0.1 \pm 0.0$ & $0.1 \pm 0.0$ \\
\hline & Untreated & $0.8 \pm 0.4$ & $0.4 \pm 0.2$ & $0.2 \pm 0.2$ \\
\hline \multirow[t]{3}{*}{ Macrophages \& giant cells } & $\mathrm{CaCl}_{2}$ & $0.1 \pm 0.0$ & $1.2 \pm 0.3$ & $0.7 \pm 0.3$ \\
\hline & $\mathrm{NaCl}$ & $0.1 \pm 0.0$ & $1.0 \pm 0.0$ & $1.7 \pm 0.6$ \\
\hline & Untreated & $0.3 \pm 0.3$ & $1.2 \pm 0.3$ & $1.3 \pm 0.3$ \\
\hline & $\mathrm{NaCl}$ & $0.0 \pm 0.0$ & $0.1 \pm 0.0$ & $2.5 \pm 0.5^{a}$ \\
\hline & Untreated & $0.0 \pm 0.0$ & $0.5 \pm 0.5$ & $1.0 \pm 0.5^{a}$ \\
\hline \multirow[t]{3}{*}{ Degradation } & $\mathrm{CaCl}_{2}$ & $0.0 \pm 0.0$ & $0.1 \pm 0.0$ & $0.4 \pm 0.2^{a}$ \\
\hline & $\mathrm{NaCl}$ & $0.0 \pm 0.0$ & $0.5 \pm 0.0$ & $2.3 \pm 1.2^{a}$ \\
\hline & Untreated & $0.0 \pm 0.0$ & $0.4 \pm 0.2$ & $1.2 \pm 0.3^{a}$ \\
\hline
\end{tabular}

${ }^{a}$ Significant differences by Kruskal Wallis test between scaffolds at one time point. Cellular influx day $3 p=0.030$; cellular influx day $90 p=0.029$; blood vessels day $90 p=0.031$; degradation day $90 p=0.044$. 


\section{Discussion}

Over the years, methods to enhance stiffness of insoluble type I collagen scaffolds have been widely investigated. Generally, crosslinking techniques are applied to stabilize collagen scaffolds, which increase their overall strength, cohesion and stiffness. ${ }^{9}$ However, for a number of applications this is not sufficient and (synthetic) polymers have been added to further improve mechanical properties. ${ }^{18}$ Another option to strengthen collagen scaffolds is to modify the collagen by the addition of methacrylates that can be set by photopolymerization, which enhances the compressive modulus of $e . g$. collagen-based gels. ${ }^{19}$ In this study, a simple method is described to adapt the stiffness of collagen scaffolds and increase their strength without modifying the base material. The method is based on treatment with a concentrated salt solution resulting in major shrinkage of the scaffolds, thus increasing the collagen concentration in the walls of the porous constructs. The method resulted in rigid collagen scaffolds that were more than 10 times stiffer than untreated scaffolds, while in vivo biocompatibility was maintained. By adjusting the type and concentration of the salt used, the method may allow the construction of scaffolds with a specific stiffness. This is relevant since every tissue in the human body has its own characteristic stiffness caused by a distinct composition of the extracellular matrix. In recent years it has become clear that stiffness of the extracellular matrix plays an important role in differentiation of stem cells. ${ }^{20}$ Consequently, to achieve optimal tissue regeneration it is important to take the stiffness of the target tissue into account when designing biomaterials. Current applications of collagen scaffolds are often limited to soft tissues, e.g. skin. Scaffolds treated with the shrinking method showed to have an average stiffness of $7.4 \times 10^{3} \mathrm{~Pa}$ with peak values of $3.0 \times 10^{4} \mathrm{~Pa}$. Consequently, this may expand the application range of collagen scaffolds to harder tissues such as muscle $(\sim 7-18 \mathrm{kPa})$, cartilage $(\sim 25 \mathrm{kPa})$ and unmineralized bone $(\sim 35 \mathrm{kPa}){ }^{21,22}$

To investigate the molecular mechanism responsible for the shrinkage, the effect of salts from the Hofmeister series on collagen scaffolds was analyzed. The influence on collagen behavior of ions from the Hofmeister series has been reported (e.g. Lim, Komsa-Penkova et al. and Rabinovich). ${ }^{23-26}$ For instance, it has been observed for telopeptide-poor type I collagen molecules at low $\mathrm{pH}$ that kosmotropic ions induce fibrous aggregates and elastic 3D networks, whereas chaotropic ions result in finer precipitates. ${ }^{26}$ In ours study, only highly concentrated salts with divalent cations $\left(\mathrm{Ca}^{2+}, \mathrm{Mg}^{2+}\right.$ and $\mathrm{Ba}^{2+}$, all at the chaotropic site of the series) in combination with an anion (not being sulfate) were able to induce shrinkage up to $90 \%$ of the initial surface area. Electron microscopical images of shrunken collagen scaffolds showed that fibrils were swollen and had lost their characteristic banding pattern, which is in line with earlier observations that $\mathrm{CaCl}_{2}$ destabilizes both the fibrillar and triple helical structure of collagen. ${ }^{27} \mathrm{~A}$ phenomenon described in leather research as 'lyotropic swelling', may explain the shrinkage. ${ }^{28}$ During lyotropic swelling, cations such as $\mathrm{Ca}^{2+}$ disrupt the hydrogen bonds that stabilize the triple helical structure. The cations interact with negatively charged carboxylic groups, thereby attracting water inside the helix, causing it to swell. For collagen, we propose the mechanism displayed in Fig. 6. Type I collagen molecules consist of $2 \alpha_{1}$ polypeptide chains and $1 \alpha_{2}$ chain, which are very close together (triple helix conformation) and are shifted one amino acid with respect to each other. This brings the carboxylic groups of the Glu and Asp amino acid residues in e.g. two $\alpha_{1}$ chains $(\sim 100$ Glu/Asp per chain) in close proximity to each other. Bivalent cations such as $\mathrm{Ca}^{2+}$ bind very well to two closely appositioned carboxylic groups, and this may be an initiating event in the denaturation of the triple helix and the subsequent swelling process. This mechanism would be different from reversible swelling by acid treatment (e.g. $0.25 \mathrm{M}$ acetic acid at $\mathrm{pH} 2.5)$ where molecules/microfibrils start to repel each other due to similarity in charge caused by the $\mathrm{pH}$. Lyotropic swelling takes place inside the triple helix and is irreversible. ${ }^{29}$ When triple helices are exposed to high concentrations of cations such as $\mathrm{Ca}^{2+}$, the triple helix gets disrupted (Fig. 6A, upper panel) causing the helix to swell. As a consequence, additional $\mathrm{Ca}^{2+}$ can enter the inner core of the helix and disrupt the hydrogen bonds that stabilize the helix structure resulting in (partial) denaturation of the triple helix. However, the (natural) covalent bonds between the individual triple helices are not affected (Fig. 6A, middle panel), preventing the fibril to fall apart. Consequently, as the helices swell, they become thicker and shorter (Fig. 6A, lower panel). On the level of fibrils, this leads to swollen but shortened fibrils (Fig. 6B). When this occurs throughout the scaffold it results in increased pore wall thickness and reduced pore size, ultimately leading to shrinkage and stiffening of the entire scaffold.

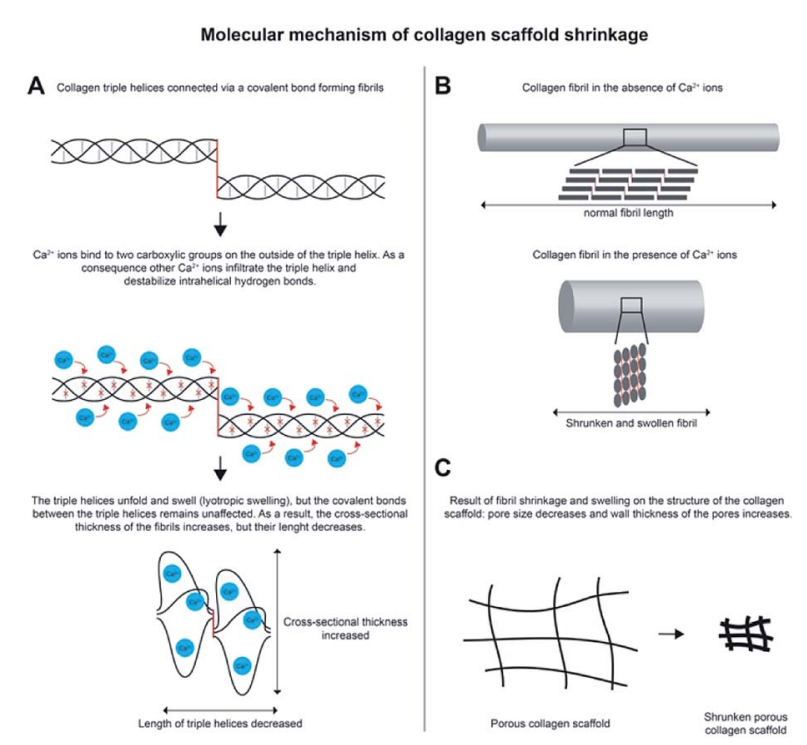

Fig. 6 Schematic representation of proposed molecular mechanism of shrinkage. (A) $\mathrm{Ca}^{2+}$ ions destabilize the triple helix by first binding to carboxylic groups and secondly by disrupting the hydrogen bonds inside the triple helix resulting in swelling of the triple helix, making it thicker but shorter. (B) The fibril structure changes dramatically after addition of $\mathrm{Ca}^{2+}$ ions: they become thicker and shorter. (C) The thicker and shorter fibrils result in shrinkage of the collagen scaffold. 
Type I collagen has shown to be a biocompatible scaffolding material in many studies, also in combination with EDC/NHS crosslinking. ${ }^{30,31}$ After $\mathrm{CaCl}_{2}$ treatment the collagen triple helix was partly denaturated as indicated by a decrease in denaturation temperature and the appearance of pepsin-derived fragments on SDS-PAGE. However, subcutaneous implantations of the $\mathrm{CaCl}_{2}$ treated scaffold revealed a similar cellular response as observed for $\mathrm{H}_{2} \mathrm{O}$ and $\mathrm{NaCl}$ treated scaffolds indicating proper biocompatibility. The major difference was that the $\mathrm{CaCl}_{2}$ treated scaffolds were barely infiltrated by cells explaining that the scaffolds were still looked intact 90 days after implantation maintaining their morphology. Biodegradability was clearly decreased and this may open ways to prepare collagen-based scaffold with long-term integrity.

The method developed to stiffen collagen scaffolds opens new opportunities in the field of regenerative medicine. By manipulating a number of parameters such as collagen concentration, $\mathrm{CaCl}_{2}$ concentration or shrinkage time, scaffolds with varying stiffness may be created. The preparation of such a wide stiffness range may serve as an in vitro platform for stem cell research to further investigate the role of stiffness on stem cell differentiation in a natural 3D extracellular matrix. Currently, this role is generally evaluated using synthetic hydrogels prepared from e.g. polyacrylamide..$^{32-34} \mathrm{~A} 3 \mathrm{D}$ environment created from a natural component of the human extracellular matrix would resemble the native stem cell niche more closely and may be advantageous to further elucidate the molecular mechanism involved in tissue regeneration and stem cell differentiation.

The in vivo results indicated that 90 days after implantation the $\mathrm{CaCl}_{2}$ treated scaffolds were not deformed by surrounding tissue, and hardly populated by cells in contrast to untreated scaffolds. The $\mathrm{CaCl}_{2}$ treated scaffolds seemed to act as a very slow degrading implant maintaining their morphological and mechanical properties over a long time period. In this respect, a function as a reservoir facilitating sustained release of drugs (e.g. anti-conception) or antigens (immunization) may be anticipated. The biocompatibility in combination with the slow degradation properties of $\mathrm{CaCl}_{2}$ treated scaffolds may be interesting as a coating for medical implants such as glucose sensors as shown in a study by Ju et al. $^{35}$

\section{Conclusions}

A method was developed to enhance stiffness of porous scaffolds prepared from type I collagen applying concentrated salt solutions, as exemplified by $\mathrm{CaCl}_{2}$. The observed shrinking effect, likely driven by the disruption of stabilizing hydrogen bonds within the triple helix, resulted in stiffer microporous scaffolds which were biocompatible, showing reduced biodegradability compared to control scaffolds. The method may be useful for the construction of collagen scaffolds with stiffness in line with the tissue to be regenerated, but may also be applied for other applications like a drug delivery system.

\section{Conflicts of interest}

There are no conflicts to declare.

\section{Acknowledgements}

The technicians from the Central Animal Laboratory, Radboud university medical center are acknowledged for their assistance with the animal experiments. Patricia Hoekstra (Marel Townsend Further Processing) is acknowledged for support with DSC measurements and analysis of DSC data. This project was financed by PIDON (NovioTissue project), a subsidiary program of the Dutch Ministry of Economic Affairs and the states of Gelderland and Overijssel (PID101020).

\section{Notes and references}

1 S. Gomes, I. B. Leonor, J. F. Mano, R. L. Reis and D. L. Kaplan, Prog. Polym. Sci., 2012, 37, 1-17.

2 A. Sorushanova, L. M. Delgado, Z. Wu, N. Shologu, A. Kshirsagar, R. Raghunath, A. M. Mullen, Y. Bayon, A. Pandit, M. Raghunath and D. I. Zeugolis, Adv. Mater., 2019, 31, e1801651.

3 A. A. Chaudhari, K. Vig, D. R. Baganizi, R. Sahu, S. Dixit, V. Dennis, S. R. Singh and S. R. Pillai, Int. J. Mol. Sci., 2016, 17(12), E1974.

4 E. A. Makris, A. H. Gomoll, K. N. Malizos, J. C. Hu and K. A. Athanasiou, Nat. Rev. Rheumatol., 2015, 11, 21-34.

5 L. R. M. Versteegden, P. de Jonge, J. IntHout, T. H. van Kuppevelt, E. Oosterwijk, W. F. J. Feitz, R. B. M. de Vries and W. F. Daamen, Eur. Urol., 2017, 72, 594-606.

6 A. George and S. Ravindran, Nano Today, 2010, 5, 254-266.

7 K. M. Brouwer, W. F. Daamen, H. R. Hoogenkamp, P. J. Geutjes, I. de Blaauw, W. Janssen-Kessels, W. de Boode, E. Versteeg, R. M. Wijnen, W. F. Feitz, M. Wijnen and T. H. van Kuppevelt, J. Biomed. Mater. Res., Part B, 2014, 102, 756-763.

8 G. P. Huang, S. Shanmugasundaram, P. Masih, D. Pandya, S. Amara, G. Collins and T. L. Arinzeh, J. Biomed. Mater. Res., Part A, 2015, 103, 762-771.

9 L. H. Olde Damink, P. J. Dijkstra, M. J. van Luyn, P. B. van Wachem, P. Nieuwenhuis and J. Feijen, Biomaterials, 1996, 17, 765-773.

10 H. Lu, H. H. Oh, N. Kawazoe, K. Yamagishi and G. Chen, Sci. Technol. Adv. Mater., 2012, 13, 064210.

11 S. J. Lee, J. Liu, S. H. Oh, S. Soker, A. Atala and J. J. Yoo, Biomaterials, 2008, 29, 2891-2898.

12 S. Toosi, H. Naderi-Meshkin, F. Kalalinia, M. T. Peivandi, H. HosseinKhani, A. R. Bahrami, A. Heirani-Tabasi, M. Mirahmadi and J. Behravan, J. Biomed. Mater. Res., Part A, 2016, 104, 2020-2028.

13 C. M. Tierney, M. G. Haugh, J. Liedl, F. Mulcahy, B. Hayes and F. J. O'Brien, J. Mech. Behav. Biomed. Mater., 2009, 2, 202-209.

14 R. L. Baldwin, Biophys. J., 1996, 71, 2056-2063.

15 J. S. Pieper, A. Oosterhof, P. J. Dijkstra, J. H. Veerkamp and T. H. van Kuppevelt, Biomaterials, 1999, 20, 847-858. 
16 S. V. Beekmans and D. Iannuzzi, Biomed. Microdevices, 2016, 18, 15.

17 L. Buttafoco, P. Engbers-Buijtenhuijs, A. A. Poot, P. J. Dijkstra, W. F. Daamen, T. H. van Kuppevelt, I. Vermes and J. Feijen, J. Biomed. Mater. Res., Part B, 2006, 77, 357-368.

18 L. E. Freed, G. C. Engelmayr Jr, J. T. Borenstein, F. T. Moutos and F. Guilak, Adv. Mater., 2009, 21, 3410-3418.

19 J. Sun, W. Q. Xiao, Y. J. Tang, K. F. Li and H. S. Fan, Soft Matter, 2012, 8, 2398-2404.

20 K. H. Vining and D. J. Mooney, Nat. Rev. Mol. Cell Biol., 2017, 18, 728-742.

21 A. Buxboim, I. L. Ivanovska and D. E. Discher, J. Cell Sci., 2010, 123, 297-308.

22 A. J. Engler, S. Sen, H. L. Sweeney and D. E. Discher, Cell, 2006, 126, 677-689.

23 J. J. Lim, Biopolymers, 1976, 15, 2371-2383.

24 R. Komsa-Penkova, R. Koynova, G. Kostov and B. G. Tenchov, Biochim. Biophys. Acta, 1996, 1297, 171-181.

25 D. Rabinovich, The chemical reactivity control of collagen: the Hofmeister effect revisited, in World Leather, 2008, pp. 26-28.

26 A. M. Oechsle, M. Landenberger, M. Gibis, S. B. Irmscher, R. Kohlus and J. Weiss, Int. J. Biol. Macromol., 2015, 79, 518-526.
27 M. Meyer, Biomed. Eng. Online, 2019, 18, 24.

28 A. D. Covington, Tanning Chemistry: The Science of Leather, Royal Society of Chemistry, Cambridge, 2011.

29 M. Redwood, W. Hummel and J. Cooke, Tanning Chemistry: The Science of Leather, Royal Society of Chemistry, 2015.

30 N. A. Hosper, A. J. Eggink, L. A. Roelofs, R. M. Wijnen, M. J. van Luyn, R. A. Bank, M. C. Harmsen, P. J. Geutjes, W. F. Daamen, T. H. van Kuppevelt, D. M. Tiemessen, E. Oosterwijk, J. J. Crevels, W. A. Blokx, F. K. Lotgering, P. P. van den Berg and W. F. Feitz, Biomaterials, 2010, 31, 3910-3919.

31 R. Parenteau-Bareil, R. Gauvin and F. Berthod, Materials, 2010, 3, 1863-1887.

32 J. H. Wen, L. G. Vincent, A. Fuhrmann, Y. S. Choi, K. C. Hribar, H. Taylor-Weiner, S. Chen and A. J. Engler, Nat. Mater., 2014, 13, 979-987.

33 J. S. Park, J. S. Chu, A. D. Tsou, R. Diop, Z. Tang, A. Wang and S. Li, Biomaterials, 2011, 32, 3921-3930.

34 W. J. Hadden, J. L. Young, A. W. Holle, M. L. McFetridge, D. Y. Kim, P. Wijesinghe, H. Taylor-Weiner, J. H. Wen, A. R. Lee, K. Bieback, B. N. Vo, D. D. Sampson, B. F. Kennedy, J. P. Spatz, A. J. Engler and Y. S. Choi, Proc. Natl. Acad. Sci. U. S. A., 2017, 114, 5647-5652.

35 Y. M. Ju, B. Yu, T. J. Koob, Y. Moussy and F. Moussy, J. Biomed. Mater. Res., Part A, 2008, 87, 136-146. 